\title{
ARTIGOS
}

\section{CORRELAÇÃO SOROLÓGICA ELETROCARDIOGRÁFICA PARA A DOENÇA DE CHAGAS EM POPULAÇÕES RURAIS NÃO SELECIONADAS DO RIO GRANDE DO SUL}

\begin{abstract}
Giovanni Baruffa, Alcino Alcantara Filho e José Osimo de Aquino Neto
$O$ trabalho analisa 4.758 correlações sorológicas eletrocardiográficas feitas em populações rurais não selecionadas de 17 municipios da Zona Sul do Rio Grande do Sul. A sorologia baseou-se na reação de fixação de complemento para doença de Chagas e os ECG foram interpretados segundo critérios da American Heart Association. 330 ECG alterados pertenciam aos 803 individuos soropositivos $(41,1 \%)$ e 1.287 aos 3.955 soronegativos (32,5\%). O gradiente de $8,5 \%$ a favor dos positivos mostrou-se significativo a nivel de $p<0,001$.

As alterações acompanhadas por diferenças significativas entre positivos e negativos foram justamente aquelas consideradas caracteristicas de miocardiopatia chagásica (BCRD, HBDAE, extrassistolia ventricular, alterações de ST e T e áreas de necrose elou fibrose. Estes fatos permitem concluir que a infecção pelo Trypanosoma cruzi é fator importante de agressão miocárdica numa parcela significativa da população rural da área endêmica de doença de Chagas no Rio Grande do Sul.
\end{abstract}

Palavras chaves: Doença de Chagas. Sorologia. ECG. População rural. Rio Grande do Sul.

O papel da infecção por Trypanosoma cruzi como fator de alterações eletrocardiográficas e cardiopatia no Rio Grande do Sul tem sido objeto de discussões desde os trabalhos de Brant ${ }^{9}$ e de Brant e cols. ${ }^{10}$. Estes autores realizaram, entre março de 1953 e agosto de 1954, um inquérito sorológico e eletrocardiográfico em populações não selecionadas dos municípios de Itaqui, Rosário do Sul, São Jerônimo e Encruzilhada do Sul e encontram $23,88 \%$ de sorologia positiva dentre 5.460 indivíduos examinados e $6,5 \%$ de eletrocardiogramas alterados em 2.172 registros. A prevalência de eletrocardiogramas alterados nos soropositivos $(5,7 \%)$, comparada com aquela encontrada nos negativos $(6,9 \%)$ demonstraria, segundo os autores, que a

Trabalho do Curso de Medicina da Universidade Católica de Pelotas, Pelotas, Rio Grande do Sul, realizado com o apoio financeiro de AZONASUL, FAPERGS: Projetos "Medicina 180/74 e 180/75" e CNPq PDE 8-004/80. Endereço para correspondência: Universidade Católica de Pelotas, Rua Felix da Cunha, 412 - 96100 Pelotas, RS, Brasil. "moléstia de Chagas nas populações estudadas do Rio Grande do Sul, não se mostra particularmente importante como fator de alterações miocárdicas crônicas"10.

Sassen e Arnt $^{26}$ em trabalho de 1959 concluem que, apesar dos altos indices de infestação triatomínica e das altas taxas de positividade sorológica na população, "a doença de Chagas não é problema médico-social de primeira grandeza no Rio Grande do Sul". Coura ${ }^{14}$ em 1966 refere como opinião corrente o fato de "que a doença de Chagas é benigna em determinadas regiōes, como no Rio Grande do Sul, e grave em outras como na Bahia e em certas áreas de Minas Gerais e Pernambuco...".

A discrepância entre nossa experiência clínica em pacientes hospitalizados ${ }^{4-6}$ e os dados da literatura, tendentes a demonstrar a escassa importância da infecção por $T$. cruzi como fator de patologia cardíaca no Rio Grande do Sul, nos levou a realizar um amplo inquérito sorológico-eletrocardiográfico, cujas correlações constituem o objeto do presente trabalho. 
Baruffa G, Alcantara Filho A, Aquino Neto J O. Correlação sorolbgica eletrocardiográfica para a doença de Chagas em populaçóes rurais nāo selecionadas do Rio Grande do Sul. Revista da Sociedade Brasileira de Medicina Tropical 16:130-138, Jul/Set, 1983

\section{MATERIAL E MÉTODOS}

O trabalho de campo foi realizado de julho de 1970 a julho de 1975 . Foram colhidas amostras de sangue e registrados eletrocardiogramas em populações rurais não selecionadas dos seguintes municípios da zona sul do Rio Grande do Sul: Canguçú, Piratini, Pedro Osório, Pinheiro Machado, Santana da Boa Vista, Caçapava, Encruzilha- da do Sul, Dom Feliciano, Camaquã, Arroio Grande, Jaguarão, Bagé, Dom Pedrito, Pelotas, São Lourenço, Lavras do Sul e Herval do Sul (Fig. 1). As características da área estudada e os.aspectos sócio-econômicos da população foram apresentados em trabalho anterior ${ }^{7}$. $O$ tamanho da amostra para cada município foi calculado de forma a representar $2 \%$ da população rural e o critério foi o de colher pequenas amostras em diferentes localidades do mesmo município.

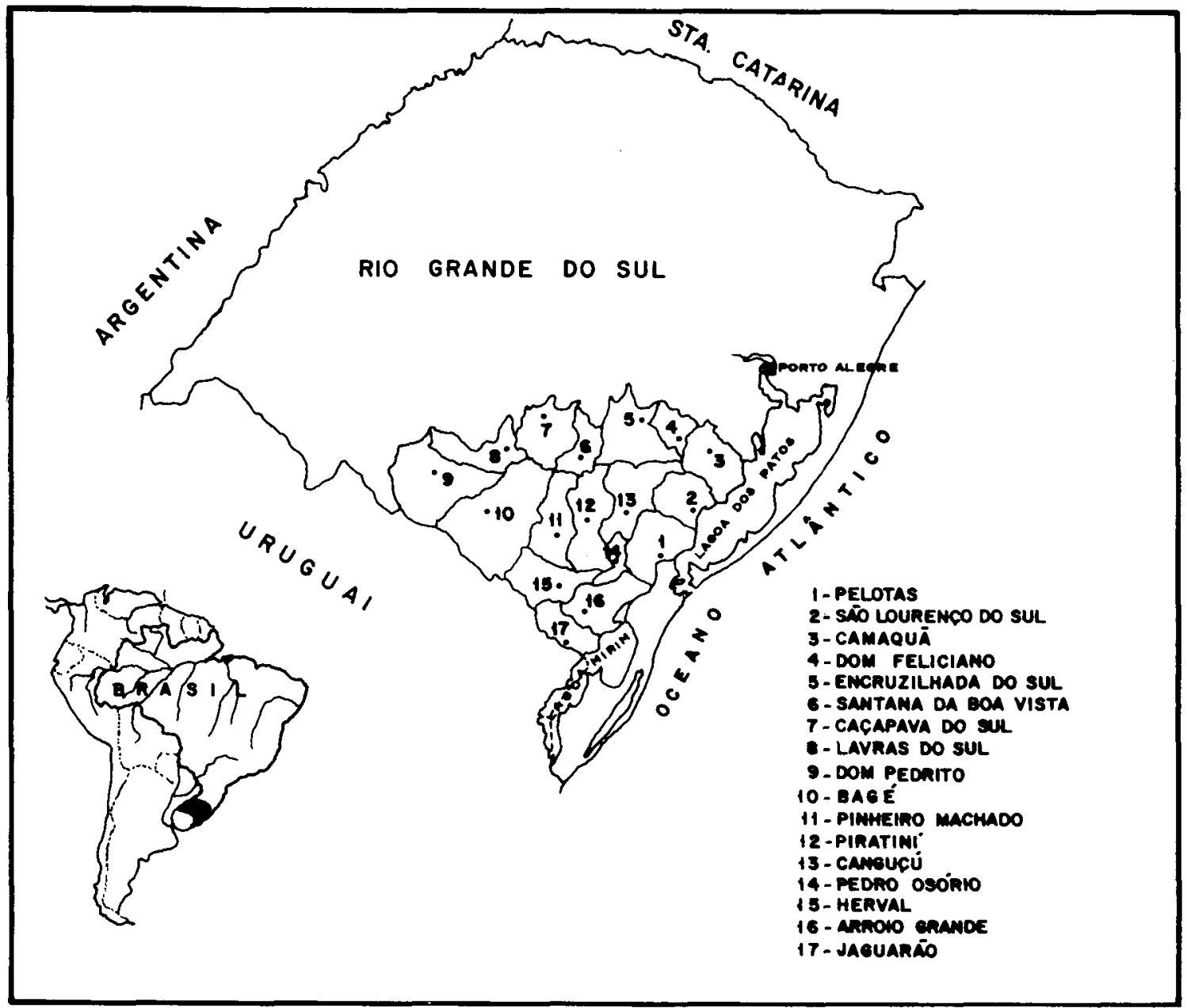

Fig. 1 - Distribuiçäo dos munıcıpıos estudados no Rio Grande do Sul e posição do Estado na America do Sul.

As reações sorológicas foram executadas parte por um de nós (AAF̣) e parte por técnico especialmente treinado no laboratório do Prof. J. O. de Almeida, da Faculdade de Medicina de Ribeirão Preto, SP. Foi desenvolvida a técnica de fixação de complemento em placa segundo Almei$\mathrm{da}^{1}$ e usado antígeno metílico de $T$. cruzi segundo
Baracchini e cols. ${ }^{3}$, preparado pelo firma Lio-Serum de Ribeirão Preto, SP.

Todos os eletrocardiogramas foram registrados por um de nós (GB) utilizando um aparelho Cardiostat $\mathrm{T}$ Siemens, alimentado com bateria de 12 volts. Foram registradas as 6 derivações do pla- 
Baruffa $G$, Alcantara Filho A, Aquino Neto J O. Correlação sorológica eletrocardiográfica para a doença de Chagas em populaçōes rurais não selecionadas do Rio Grande do Sul. Revista da Sociedade Brasileira de Medicina Tropical 16:130-138, Jul/Set, 1983

no frontal e as 6 precordiais. Para facilitar a leitura e interpretação foram anotadas nos traçados: sexo, idade, pressão arterial $\mathrm{e}$, nas mulheres, eventual presença e período de gravidez. A interpretação, realizada sem conhecimento prévio dos resultados da sorologia, ficou a cargo de um de nós (JOAN) que seguiu os critérios da American Heart Association.

As alterações eletrocardiográficas foram classificadas e agrupadas como se segue:

19) alterações de formação de estímulo: taquicardia sinusal (freqüência acima de $100 \mathrm{bpm}$ ), bradicardia sinusal (abaixo de $60 \mathrm{bpm}$ ), extrassístoles supraventriculares, extrassístoles ventriculares unifocais e/ou polifocais, flutter e fibrilação atrial, ritmo nodal;

29) alterações de condução do estímulo: bloqueios $\mathrm{A}-\mathrm{V}$, bloqueios de ramos incompletos ou completos, distúrbio da condução intraventricular;

$3^{\circ}$ ) alterações de ST e T;

4\%) sobrecarga de cavidades;
59) alterações sugestivas de necrose e/ou fibrose;

69) baixa voltagem do QRS.

A avaliação da significância das diferenças entre as porcentagens foi realizada empregando-se o teste de $\mathrm{X}^{2}$, sendo estipulado o nivel de significância de $\mathrm{p}<0,05$.

\section{RESULTADOS}

Foram aproveitadas no inquérito 5.530 amostras de soro, das quais 973 deram a reação de fixação de complemento positiva $(17,59 \%)$. Os dados completos do inquérito sorológico foram publicados anteriormente ${ }^{7}$.

Foram registrados 4.758 eletrocardiogramas, sendo 803 em indivíduos soropositivos e $3.955 \mathrm{em}$ soronegativos. A distribuição dos eletrocardiogramas por grupo etário e positividade sorológica estão representadas na Tabela 1 . Na Figura 2 é apresentada a distribuição da amostra por grupo etário e por sexo.

Tabela 1 -Distribuição dos ECG registrados por gnupo etário e positividade

\begin{tabular}{|c|c|c|c|c|c|}
\hline \multirow{2}{*}{$\begin{array}{l}\text { Grupo } \\
\text { Etário }\end{array}$} & \multicolumn{3}{|c|}{ Sorologia } & \multicolumn{2}{|c|}{ Total } \\
\hline & $\begin{array}{c}\text { Negativo } \\
N o\end{array}$ & $N O$ & $\ddot{\%}$ & $N ?$ & $\%$ Amostra \\
\hline $\begin{array}{r}5-9 \\
10-19 \\
20-29 \\
30-39 \\
40-49 \\
50-59 \\
60-69 \\
70 \mathrm{e}+\end{array}$ & $\begin{array}{r}81 \\
732 \\
695 \\
788 \\
744 \\
508 \\
263 \\
144\end{array}$ & $\begin{array}{r}7 \\
82 \\
121 \\
146 \\
178 \\
160 \\
83 \\
26\end{array}$ & $\begin{array}{r}7,95 \\
10,07 \\
14,82 \\
15,63 \\
19,30 \\
23,95 \\
23,98 \\
15,29\end{array}$ & $\begin{array}{r}88 \\
814 \\
816 \\
934 \\
922 \\
668 \\
346 \\
170\end{array}$ & $\begin{array}{r}1,85 \\
17,10 \\
17,15 \\
19,63 \\
19,37 \\
14,03 \\
7,27 \\
3,57\end{array}$ \\
\hline Total & 3.955 & 803 & 16,87 & 4.758 & 100,00 \\
\hline
\end{tabular}


Baruffa G, Alcantara Filho A, Aquino Neto J O. Correlação sorológica eletrocardiográfica para a doença de Chagas em populą̧̃es rurais não selecionadas do Rio Grande do Sul. Revista da Sociedade Brasileira de Medicina Tropical 16:130-138, Jul/Set, 1983



Fig. 2 - Distribuição dos 4.758 ECG na amostra: os números acima das barras indicam os ECG registrados no grupo etário respectivo. As áreas hachureadas e os números imediatamente acima delas representam os masculinos de cada grupo etário. A abscissa indica a percentagem com que cada grupo etário contribui na amostra total.

Apresentaram-se alterados, de acordo com os critérios mencionados em material e métodos, 1.617 eletrocardiogramas $(34,0 \%)$, sendo 330 nos soropositivos (41 1\%) e 1.287 nos soronegativos $(32,5 \%)$. A análise estatística mostrou que esta diferença é significativa a nível de $\mathrm{p}<0,001\left(\mathrm{X}^{2}\right.$ $=21,68$ ).

Consignamos na Tabela 2 o número de ele- trocardiogramas alterados e as alterações detectadas nestes traçados, de acordo com a reatividade sorológica. Nos positivos tivemos 531 alterações em 330 traçados alterados, o que dá uma média de 1,60 alterações por traçado. Nos negativos encontramos 1.664 alterações em 1.287 traçados alterados, dando uma média de 1,29 alterações por traçado. A diferença mostrou-se significativa a nivel de $\mathrm{p}<0,001\left(\mathrm{X}^{2}=21,68\right)$. 
Baruffa G, Alcantara Filho A, Aquino Neto J O. Correlação sorológica eletrocardiográfica para a doença de Chagas em populaçöes rurais não selecionadas do Rio Grande do Sul. Revista da Sociedade Brasileira de Medicina Tropical 16:130-138, Jul/Set, 1983

Tabela 2 - Alterações electrocardiográficas em relação à reatividade sorológica para Trypanosoma cruzi .

\begin{tabular}{|c|c|c|c|c|c|c|c|c|}
\hline \multirow{2}{*}{$\begin{array}{l}\text { Alterações } \\
\text { Eletrocardiográficas }\end{array}$} & \multicolumn{2}{|c|}{$\begin{array}{c}\text { Positivos } \\
803\end{array}$} & \multicolumn{2}{|c|}{$\begin{array}{l}\text { Negativos } \\
3.955\end{array}$} & \multicolumn{2}{|c|}{$\begin{array}{l}\text { Total } \\
4.758\end{array}$} & \multicolumn{2}{|c|}{ Significância } \\
\hline & $N Q$ & $\%$ & $N ?$ & $\%$ & $N o$ & $\%$ & $X^{2}$ & $P<$ \\
\hline Traçados alterados & 330 & 41,09 & 1.287 & 32,54 & 1.617 & 33,98 & 21,75 & 0,001 \\
\hline Taquicardia sinusal & 74 & 9,21 & 302 & 7,63 & 376 & 7,90 & 2,26 & $\mathrm{~N} / \mathrm{S}$ \\
\hline Bradicardia sinusal & 32 & 3,98 & 111 & 2,80 & 143 & 3,00 & 3,15 & $\mathrm{~N} / \mathrm{S}$ \\
\hline Extra. supra. ventr. & 23 & 2,86 & 46 & 1,16 & 69 & 1,45 & 13,53 & 0,001 \\
\hline Extra. ventr. unif. & 24 & 2,98 & 121 & 3,05 & 145 & 3,04 & 0,02 & $\mathrm{~N} / \mathrm{S}$ \\
\hline Extra. ventr. pol. & 19 & 2,36 & 26 & 0,65 & 45 & 0,94 & 20,76 & 0,001 \\
\hline Fibrilação atrial & 2 & 0,24 & 8 & 0,20 & 10 & 0,21 & 0,60 & $\mathrm{~N} / \mathrm{S}$ \\
\hline Ritmo nodal & 5 & 0,62 & 10 & 0,25 & 15 & 0,31 & 2,90 & $\mathrm{~N} / \mathrm{S}$ \\
\hline Bloqueio A-V 19 grau & 3 & 0,37 & 16 & 0,40 & 19 & 0,40 & 0,01 & $\mathrm{~N} / \mathrm{S}$ \\
\hline Bloqueio A-V 20 grau & 2 & 0,24 & - & - & 2 & 0,04 & 8,58 & 0,01 \\
\hline Bloqueio A-V 39 grau & - & - & - & - & - & - & - & - \\
\hline B.I.R.D. & 7 & 0,87 & 27 & 0,68 & 34 & 0,71 & 0,32 & $\mathrm{~N} / \mathrm{S}$ \\
\hline H.B.A.E. & 58 & 7,22 & 129 & 3,26 & 117 & 3,93 & 27,69 & 0,001 \\
\hline B.C.R.D. & 29 & 3,61 & 33 & 0,93 & 62 & 1,30 & 39,83 & 0,001 \\
\hline B C.R.E. & 3 & 0,37 & 7 & 0,17 & 10 & 0,21 & 1,20 & $\mathrm{~N} / \mathrm{S}$ \\
\hline Dist. Cond. Intrav. & 8 & 0,99 & 43 & 1,08 & 51 & 1,07 & 0,04 & $\mathrm{~N} / \mathrm{S}$ \\
\hline Alterações de ST e T & 146 & 18,18 & 489 & 12,36 & 635 & 13,34 & 19,48 & 0,001 \\
\hline Sobrecarga atrial D & - & - & 4 & 0,10 & 4 & 0,08 & 0,77 & $\mathrm{~N} / \mathrm{S}$ \\
\hline Sobrecarga atrial $\mathrm{E}$ & 6 & 0,74 & 52 & 1,31 & 58 & 1,21 & 1,74 & $\mathrm{~N} / \mathrm{S}$ \\
\hline Sobrecarga ventr. D & 7 & 0,87 & 25 & 0,63 & 32 & 0,67 & 0,55 & $\mathrm{~N} / \mathrm{S}$ \\
\hline Sobrecarga ventr. E & 40 & 4,98 & 84 & 2,12 & 124 & 2,60 & 21,29 & 0,001 \\
\hline Necrose e/ou fibrose & 33 & 4,10 & 85 & 2,14 & 118 & 2,48 & 10,49 & 0,01 \\
\hline Baixa voltagem de QRS & 10 & 1,24 & 46 & 1,16 & 56 & 1,17 & 0,03 & $\mathrm{~N} / \mathrm{S}$ \\
\hline
\end{tabular}

A amostra apresenta uma diferença significativa $(\mathrm{p}<0,001)$ entre a positividade sorológica nos homens $(18,96 \%)$ e nas mulheres $(15,03 \%)$. Entretanto as mulheres no total apresentam uma porcentagem de alterações eletrocardiográficas significativamente maior que os homens: $35,30 \%$ ver- sus 32,49\%: $\mathrm{p}<0,05\left(\mathrm{X}^{2}=4,13\right)$. A Tabela 3 mostra que a significância se refere às alterações nos negativos de ambos os sexos, não havendo diferença significativa entre homens e mulheres soropositivos.

Tabela 3 - Distribuição dos ECG registrados (Reg.) e dos alterados (Alt.) em relação ao sexo e reatividade sorológica e significância das diferenças observadas

ECG Total 4.758

\begin{tabular}{|c|c|c|c|c|c|c|c|c|}
\hline \multirow{2}{*}{ Sorologia } & \multicolumn{3}{|c|}{ Homens } & \multicolumn{3}{|c|}{ Mulheres } & \multicolumn{2}{|c|}{ Significância } \\
\hline & Reg. & Alt. & $\%$ & Reg. & Alt. & $\%$ & $X^{2}$ & $P<$ \\
\hline $\begin{array}{l}\text { Positivo } \\
\text { Negativo }\end{array}$ & $\begin{array}{r}432 \\
1.808\end{array}$ & $\begin{array}{l}167 \\
558\end{array}$ & $\begin{array}{l}39,47 \\
30,86\end{array}$ & $\begin{array}{r}380 \\
2.147\end{array}$ & $\begin{array}{l}163 \\
729\end{array}$ & $\begin{array}{l}42,89 \\
33,95\end{array}$ & $\begin{array}{l}0,93 \\
4,25\end{array}$ & $\begin{array}{l}N / S \\
0,05\end{array}$ \\
\hline Total & 2.231 & 725 & 32,49 & 2.527 & 892 & 35,30 & 4,13 & 0,05 \\
\hline
\end{tabular}


Baruffa G, Alcantara Filho A, Aquino Neto J O. Correlação sorológica eletrocardiográfica para a doença de Chagas em populaçóes rurais não selecionadas do Rio Grande do Sul. Revista da Sociedade Brasileira de Medicina Tropical 16:130.138, Jul/Set, 1983

Tabela 4 - Alteraçōes eletrocardiográficas que apresentaram diferenças estatisticamente significativas com relação à reatividade sorológica nos dois sexos

\begin{tabular}{|c|c|c|c|c|c|c|c|c|c|c|c|c|}
\hline \multirow{3}{*}{$\begin{array}{l}\text { Alteraçōes } \\
\text { Eletrocardiográficas }\end{array}$} & \multicolumn{6}{|c|}{ Homens: 2.231} & \multicolumn{6}{|c|}{ Mulheres 2.527} \\
\hline & \multirow{2}{*}{$\frac{\text { Positivos: }}{\text { No }}$} & \multirow{2}{*}{$\frac{423}{\%}$} & \multirow{2}{*}{$\frac{\text { Negativos: }}{\text { No }}$} & \multirow{2}{*}{$\frac{1808}{\%}$} & \multicolumn{2}{|c|}{ Significância } & \multirow{2}{*}{$\frac{\text { Positivos: }}{N^{O}}$} & \multirow{2}{*}{$\frac{380}{\%}$} & \multirow{2}{*}{$\frac{\text { Negativos: }}{N o}$} & \multirow{2}{*}{$\frac{2.147}{\%}$} & \multicolumn{2}{|c|}{ Significância } \\
\hline & & & & & $X^{2}$ & $P<$ & & & & & $X^{2}$ & $P<$ \\
\hline Bradicardia sinusal & 26 & 6,14 & 102 & 5,64 & 0,14 & $N / S$ & 6 & 1,57 & 9 & 0,41 & 7,35 & 0,01 \\
\hline E.S.V. & 12 & 2,83 & 21 & 1,16 & 6,44 & 0,02 & 11 & 2,89 & 25 & 1,16 & 6,58 & 0,02 \\
\hline E.VP. & 9 & 2,12 & 9 & 0,40 & 10,83 & 0,001 & 10 & 2,63 & 17 & 0,67 & 10,10 & 0,01 \\
\hline Bloqueio A.V $2 \circ$ grau & 1 & 0,23 & - & - & 4,26 & 0,05 & 1 & 0.26 & - & - & 5,66 & 0,05 \\
\hline B.C.R.D. & 20 & 4,73 & 24 & 1,32 & 20,17 & 0,001 & 9 & 2,36 & 9 & 0,42 & 17,40 & 0,001 \\
\hline H.B.A.E. & 45 & 10,63 & 75 & 4,14 & 28,18 & 0,001 & 13 & 3,42 & 54 & 2,51 & 0,99 & $N / S$ \\
\hline Alteração de ST e T & 62 & 14,65 & 162 & 8,96 & 12,26 & 0,001 & 84 & 22,10 & 327 & 15,23 & 11,18 & 0,001 \\
\hline S.V.E. & 22 & 5,2 & 49 & 2,71 & 6,81 & 0,01 & 18 & 4,73 & 35 & 1,63 & 15,02 & 0,001 \\
\hline Necrose/fibrose & 23 & 5,43 & 40 & 2,21 & 12,80 & 0,001 & 10 & 2,63 & 45 & 2,09 & 0,40 & $\mathrm{~N} / \mathrm{S}$ \\
\hline
\end{tabular}

Na Tabela 4 são consideradas as alterações eletrocardiográficas que mostram diferença significativa entre positivos e negativos nos dois sexos.
Na Tabela 5 estão reagrupadas as alterações eletrocardiográficas mais significativas levando-se em conta os grupos de idade abaixo e acima de 50 anos.

Tabela 5 - Alteraçōes eletrocardiográficas que apresentam differenças estatisticamente significativa com relaçäo à reatividade sorológica nos grupos etários abaixo e acima dos 50 anos

\begin{tabular}{|c|c|c|c|c|c|c|c|c|c|c|c|c|c|}
\hline \multirow{3}{*}{$\begin{array}{l}\text { Alteraçōes } \\
\text { Eletrocardiográficas }\end{array}$} & \multicolumn{6}{|c|}{$<50$ anos: 3.574} & \multirow{3}{*}{. } & \multicolumn{6}{|c|}{$\geqslant 50$ anos: 1.184} \\
\hline & \multirow{2}{*}{$\frac{\text { Positivos: }}{N o}$} & \multirow{2}{*}{$\frac{534}{\%}$} & \multirow{2}{*}{$\frac{\text { Negativos: }}{N ?}$} & \multirow{2}{*}{$\frac{3.040}{\%}$} & \multicolumn{2}{|c|}{ Significância } & & \multirow{2}{*}{$\frac{\text { Positivos: }}{N^{O}}$} & \multirow{2}{*}{$\frac{269}{\%}$} & \multirow{2}{*}{$\frac{\text { Negativos: }}{N O}$} & \multirow{2}{*}{$\frac{915}{\%}$} & \multicolumn{2}{|c|}{ Significância } \\
\hline & & & & & $X^{2}$ & $P<$ & & & & & & $X^{2}$ & $P<$ \\
\hline E.S.V. & 10 & 1,87 & 27 & 0,88 & 4,35 & 0,05 & & 13 & 4,83 & 19 & 2,07 & 5,62 & 0,05 \\
\hline E.V.P. & 3 & 0,56 & 12 & 0,39 & 0,29 & $\mathrm{~N} / \mathrm{S}$ & & 16 & 5,94 & 14 & 1,53 & 16,49 & 0,001 \\
\hline B.A.V. $2^{\circ} \mathrm{grau}$ & 2 & 0,37 & - & - & 11,30 & 0,001 & & - & - & - & - & - & - \\
\hline B.C.R.D. & 11 & 2,05 & 13 & 0,42 & 17,84 & 0,001 & & 18 & 6,69 & 20 & 2,18 & 622,14 & 0,001 \\
\hline H.B.A.E. & 26 & 4,86 & 53 & 1,74 & 20,52 & 0,001 & & 32 & 11,89 & 76 & 8,30 & 3,22 & $\mathrm{~N} / \mathrm{S}$ \\
\hline Alteração de T e ST & 66 & 12,35 & 268 & 8,81 & 6,63 & 0,01 & & 80 & 29,73 & 221 & 24,15 & 3,19 & $\mathrm{~N} / \mathrm{S}$ \\
\hline S.V.E. & 12 & 2,24 & 34 & 1,11 & 4,25 & 0,05 & & 28 & 10,40 & 50 & 5,46 & 8,09 & 0,01 \\
\hline Necrose/fibrose & 14 & 2,62 & 35 & 1,15 & 12,38 & 0,001 & & 19 & 7,06 & 50 & 5,46 & 0,94 & $\mathrm{~N} / \mathrm{S}$ \\
\hline
\end{tabular}

\section{DISCUSSÃO}

O trabalho de campo, de colheita de amostras e registros dos eletrocardiogramas, se desenvolveu no curso de 6 anos e cobriu uma área de aproximadamente $50.300 \mathrm{~km}^{2}$ com uma população estimada na época em 720.500 habitantes, dos quais $40 \%$ viviam em área rural ${ }^{71}$. A área corresponde aproximadamente a $1 / 5$ da superfície do Rio Grande do Sul (Fig. 1) e a população a mais de $1 / 10$.

Pela Tabela 1 podemos ver que o tamanho da amostra é suficientemente representativo do universo e bastante homogêneo na sua distribuição por grupos de idades. Isto é válido sobretudo para os grupos de 10 a 59 anos, considerando os mais importantes para o estudo da morbidade cardíaca na forma crônica da doença de Chagas. A Figura 2 mostra que os dois sexos são representados de forma bastante consistente em cada grupo etário, com uma discreta predominância das mulheres até 39 anos e dos homens dos 40 anos em diante.

Os trabalhos de correlação sorológica eletrocardiográfica realizados em outras áreas endêmicas de doença de Chagas do Brasil e da América do Sul, mostram uma diferença significativa nas alterações eletrocardiográficas dos soropositivos com respeito aos soronegativos. Tal diferença ou "excesso de risco" é atribuida às lesões miocárdias originadas pela infecção por $T$. cruzi ${ }^{15} 17182223$. Os dados da Tabela 2 mostram que isto acontece 
também no Rio Grande do Sul. De fato, encontramos uma elevada significância $(\mathrm{p}<0,001)$ nas diferenças de extrassístoles ventriculares polifocais, hemibloqueio anterior esquerdo, bloqueio completo do ramo direito e alterações de ST e T. Todo esse conjunto está incluído na constelação de alteraçðes eletrocardiográficas classicamente consideradas sugestivas de cardiomiopatia chagásica ${ }^{11} 19$ $23-25$.

Pode-se ainda acrescentar que a média de alterações por traçado nos soropositivos $(1,60)$ é sigficativamente superior à média encontrada nos soronegativos $(1,29)$ com $p<0,001$. Isto demonstra a tendência dos chagásicos a apresentarem alterações eletrocardiográficas múltiplas. Uma das associaçōes mais características de alterações eletrocardiográficas em chagásicos é o HBAE + BCRD que foi visto em aproximadamente $30 \%$ dos nossos registros em que havia BCRD. Esta mesma associação foi encontrada em $50 \%$ de pacientes chagásicos hospitalizados em que havia BCRD e procedentes da mesma área de estudo ${ }^{4}$.

Entretanto no trabalho de correlação sorológica eletrocardiográfica realizado em 4 municípios gaúchos, Brant e cols. ${ }^{10}$ não encontraram diferenças significativas entre percentagens de alterações nos positivos em relação aos negativos.

Baruffa $^{5}$ em trabalho análogo realizado no município de Encruzilhada do Sul, também não encontrou diferenças significativas entre alterações nos positivos e negativos. Todavia o gradiente entre as percentagens de alterações nos dois grupos foi de $8,66 \%$, praticamente igual ao encontrado no presente trabalho $(8,55 \%)$. Nesse trabalho a baixa significância está provavelmente relacionada ao reduzido tamanho da amostra: 350 registros eletrocardiográficos. Considerando-se porém uma das alterações mais sugestivas de etiologia chagásica, o $\mathrm{BCRD}$, a diferença entre positivos e negativos em Encruzilhada do Sul revela-se significativa a nivel de $\mathrm{p}<0,02\left(\mathrm{X}^{2}=5,93\right)^{5}$.

Recentemente Macêdo e cols. ${ }^{21}$ analisaram 4.982 registros eletrocardiográficos obtidos em 11 Estados brasileiros, sendo 1.546 no Rio Grande do Sul. Encontraram nestes últimos $24,5 \%$ de alteraçōes nos soropositivos e $21,5 \%$ nos soronegativos, o gradiente de $3 \%$ não mostrou-se estatisticamente significativo.

A discrepância entre os autores citados e os dados do presente trabalho pode ser atribuida a fatores como: a grande homogeneidade da nossa amostra, tomada toda em área de alta endemicidade; ao tamanho da mesma: 4.758 correlações sorológicas-eletrocardiográficas e às diferenças metodológicas na execução dos trabalhos.

A Tabela 3 analisa as alterações eletrocardiográficas nos dois sexos em relação com a reatividade sorológica. Podemos ver que as mulheres tem uma prevalência de alterações eletrocardiográficas significativamente maior que os homens $(\mathrm{p}<0,05)$. O responsável por este comportamento é o grupo soronegativo que mostra uma prevalência de alterações eletrocardiográficas significativamente maior no sexo feminino $(p<0,05)$. No grupo soropositivo, apesar da percentagem de alterações ser um pouco maior no sexo feminino $(42,89 \%$ versus $39,47 \%$ ) a diferença não mostrou-se significativa.

A ausência de uma nítida diferença de prevalência de alterações eletrocardiográficas entre os homens e mulheres soropositivos contrasta com quanto encontrado por outros autores ${ }^{11} 171923$. Todavia Correia-Lima ${ }^{13}$ em Oeiras no Piauí, encontrou maior prevalência de alterações nas mulheres e Macêdo ${ }^{20}$ não encontrou diferenças entre os dois sexos na área de São Felipe na Bahia.

A Tabela 4 mostra que as ESV, as EVP, o $\mathrm{BCRD}$ e as alterações de $\mathrm{ST}$ e $\mathrm{T}$ apresentam diferença significativa entre soropositivos e soronegativos tanto nos homens quanto nas mulheres, mostrando assim que as alterações eletrocardiográficas mais características da miocardiopatia chagásica são comuns aos dois sexos.

A Tabela 5 mostra a distribuição das alteraçōes consideradas na tabela anterior nos grupos etários abaixo e acima de 50 anos. Podemos ver que a prevalência das alterações eletrocardiográficas assinaladas na mesma apresenta um aumento global com a idade, tanto nos soropositivos quanto nos soronegativos, fato mencionado por outros au. tores $^{17} 2023$. Chama atenção que no grupo abaixo de 50 anos, com excessão das EVP, todas as demais alterações mostram prevalências com diferenças altamente significativas nos soropositivos comparados com os soronegativos. Assim com referência ao $\mathrm{BCRD}$, ao $\mathrm{HBAE}$, e as alterações sugestivas de necrose e/ou fibrose, a significância é da ordem de $\mathrm{p}<0,001$, e os únicos dois registros de BAV de IIO grau pertencem justamente 
Baruffa G, Alcantara Filho A, Aquino Neto J O. Correlaçāo sorológica eletrocardiográfica para a doença de Chagas em populaçōes rurais näo selecionadas do Rio Grande do Sul. Revista da Sociedade Brasileira de Medicina Tropical 16:130-138, $\mathrm{Jul} / \mathrm{Set}, 1983$

a pessoas soropositivas menores de 50 anos. No grupo etário acima de 50 anos a não ser o BCRD, estas diferenças já não são notadas. A explicação talvez esteja no fato que, alcançada a idade a qual se intensificam os processos degenerativos miocárdicos de origem vascular, as alterações eletrocardiográficas relacionadas a tais processos sofrem um acentuado aumento da prevalência no grupo soronegativo.

A análise dos dados expostos nos permite concluir que a correlação sorológica eletrocardiográfica para doença de Chagas na Zona Sul do Rio Grande do Sul mostra uma elevada prevalência de alterações eletrocardiográficas nos soropositivos $(41,10 \%)$ e um gradiente significativo de diferença com relação às alterações nos soronegativos ( $p<0,001)$. E mais, as alterações eletrocardiográficas qualitativas encontradas nos positivos são superponíveis àquelas encontradas em chagásicos de outras regióes do Brasil e da América ${ }^{11} 1519$ 2325 .

Todavia não é nossa intenção afirmar que a infecção chagásica no Rio Grande do Sul mostre a mesma prevalência de lesões miocárdicas das outras áreas endêmicas. A doença de Chagas não seria a mesma em todas as regiōes configurando aquilo que Brant ${ }^{8}$ definiu como "matizes regionais". Para isso concorreriam vários elementos, entre eles: a cepa de $T$. cruzi ${ }^{2}$, a possibilidade $\mathrm{e}$ freqüência de reinfecções ${ }^{20}$, o ambiente físico, social e cultural no qual se insere a endemia; fatores alimentares, ocupação, infecções concomitantes e outros ${ }^{12}$.

O que porém não nos parece aceitável é a conclusão que no Rio Grande do Sul a infecção pelo $T$. cruzi tenha um curso benigno e muito menos que seja desprovida de morbidade cardíaca. Pelo contrário, a nosso ver a doença de Chagas neste estado se constitui num sério problema médico-social, não só com relação aos pacientes hospitalizados, mas também para uma parcela significativa da população rural da área endêmica.

\section{SUMMARY}

This paper analyzes 4,758 serological-electrocardiographic correlations obtained at random in a rural population from 17 countries of the far South of Rio Grande do Sul (Brasil). Sera were examined by the complement fixation test (CFT) for Chagas' disease and the electrocardiogram
(ECG) tracings were interpreted according to the criteria of the American Heart Association. Among 803 people with a positive CFT, 330 had an altered ECG (41.1\%), among 3,955 with a negative CFT the ECG were altered in 1,287 (32.5\%). The $8.5 \%$ gradient between positive and negative results, was significant at $p<0,001$ level.

The ECG abnormalities more frequently associated with seropositivity were those often found in Chagas' cadiopathy (right branch block, left anterior branch hemiblock, ventricular premature beats, ST and T abnormalities and areas of necrosis and/or fibrosis). The authors conclude that in Rio Grande do Sul (Brasil) Chagas'disease is a very important etiologic factor of myocardial injury among people living in endemic areas.

Key words: Chagas' disease. Serology. ECG. Rural areas. Rio Grande do Sul. Brasil.

\section{AGRADECIMENTOS}

Ao Paulo Silva pela precisão do traço na confeç̧ão das ilustrações e a Dioneides C. de Oliveira pelo esmero no trabalho de datilografia.

\section{REFERÊNCIAS BIBLIOGRÁFICAS}

1. Almeida JO. Reação de fixação pela técnica quantitativa para moléstia de Chagas. Técnica em tubos e técnica em placas. In: Cançado JR (ed) Doença de Chagas. Imprensa Oficial de Minas Gerais, Belo Horizonte, p. 279-314, 1968.

2. Andrade SC. Caracterização de cepas de Trypanosoma cruzi isoladas no Recôncavo Baiano. Tese. Universidade Federal da Bahia, Salvador, 1973.

3. Baracchini O, Costa A, Carloni J. Emprego do calor e do metanol no preparo do antígeno de Trypanosoma cruzi. Hospital 68: 193-199,1965.

4. Baruffa G. Contribuição ao conhecimento da forma crônica da doença de Chagas na Zona Sul do Rio Grande do Sul. Revista da Associação Médica do Rio Grande do Sul 18:237-250, 1974.

5. Baruffa G. Prevalência sorológica da doença de Chagas e correlação sorológica-eletrocardiográfica em populações não selecionadas do Município de EncruziIhada do Sul, Rio Grande do Sul. Revista da Sociedade Brasileira de Medicina Tropical 9:95-104, 1975.

6. Baruffa G, Aquino Neto JO, Alcantara A, Oliveira de Morais JC, Barros FCLF. Manifestações cardíacas e digestivas da doença de Chagas na Zona Sul do Rio Grande do Sul. Revista Goiana de Medicina 16:189$-209,1970$.

7. Baruffa G, Alcantara A. Prevalência sorológica da 
doença de Chagas na Zona Sul do Rio Grande do Sul (Brasil). Revista do Instituto de Medicina Tropical de São Paulo 19:117-123, 1977.

8. Brant TC. Matizes regionais da doença de Chagas. Revista Brasileira de Malariologia e Doenças Tropicais 18:3-8, 1966.

9. Brant TC. Razões para nova orientação nas pesquisas sobre doença de Chagas no Estado do Rio Grande do Sul. Revista Brasileira de Malariologia e Doenças Tropicais 18:105-112, 1966.

10. Brant TC, Laranja FS, Bustamante FM, Leite Mello A. Dados sorológicos e eletrocardiográficos obtidos em populações não selecionadas de zonas endêmicas de doença de Chagas no Estado do Rio Grande do Sul. Revista Brasileira de Malariologia e Doenças Tropicais 9:141-148, 1957.

11. Brasil A. Evolução e prognóstico da doença de Chagas. Arquivos Brasileiros de Cardiologia 18:365-380, 1965.

12. Conselho Nacional de Desenvolvimento Científico e Tecnológico (CNPq). Epidemiologia da doença de Chagas. Objetivos e metodologia dos estudos longitudinais. Relatório Técnico n? 1, 1974.

13. Correia Lima FG. Doença de Chagas no município de Oeiras, Piauí; estudo seccional nas localidades de Colonia e Oitis. Tese. Faculdade de Medicina da Universidade Federal do Rio de Janeiro, Rio de Janeiro, 1976.

14. Coura JR. Contribuição ao estudo da doença de Chagas no Estado da Guanabara. Revista Brasileira de Malariologia e Doenças Tropicais 18:9-98, 1966.

15. Coura JR, Annunziato N, Abreu LL, Dubois LG, Correia Lima FG. Gradiente de alterações eletrocardiográficas em grupos de indivíduos com sorologia positiva negativa para infecção chagásica, pareados por idade e sexo. In: Anais do Congresso Internacional sobre Doença de Chagas. Rio de Janeiro p. 42, 1979.
16. Demografia. Secretaria de Coordenação e Planejamento, Superintendência de Estatística e Informática. Porto Alegre Vol 1, 1972.

17. Dubois L. Morbidade da doença de Chagas. Estudo seccional de uma área endêmica. Tese. Universidade Federal do Rio de Janeiro, Rio de Janeiro, 1977.

18. Faria CAF. Condições de saúde e doença de trabalhadores rurais do município de Luz, MG, com especial atenção à prevalência da moléstia de Chagas. Tese. Faculdade de Medicina da Universidade Federal de Minas Gerais, Belo Horizonte, 1978.

19. Laranja RS, Dias E, Nóbrega G, Miranda A. Chagas' disease. A clinical, epidemiologic and pathologic study. Circulation 14:1035-1060, 1956.

20. Macêdo VO. Influência da exposição à reinfecção na evolução da doença de Chagas. Revista de Patologia Tropical 5: 33-116, 1976.

21. Macêdo V, Prata A, Rodrigues DA, Silva G, Castilhos Z. Prevalência de alterações eletrocardiográficas em chagásicos (informações preliminares sobre o inquérito eletrocardiográfico nacional). Arquivos Brasileiros de Cardiologia 38: 261-264, 1982.

22. Organização Mundial de la Salud. Aspectos clínicos de la enfermedad de Chagas. Boletin de la Oficina Sanitária Panamericana 77:141-158, 1974.

23. Puigbó JS, Nava Rhode JR, Garcia Barrios H, Suares JA, Gil Yépez C. Clinical and epidemiological study of chronic heart involvement in Chagas' disease. Bulletin of the World Health Organization 34:655-669, 1966.

24. Prata A. Natural history of chagasic cardiomiopathy. PAHO Scientific Publication 318:191-193, 1976.

25. Rosembaum MB, Alvarez AJ. The electrocardiogram in chronic chagasic myocarditis. American Heart Journal 50:492-527, 1955.

26. Sassen FA, Arnt LC. A doença de Chagas no Rio Grande do Sul. Revista de Medicina do Rio Grande do Sul 15:133-142, 1959. 